http://dx.doi.org/10.22402/j.rdipycs.unam.3.1.2017.56.38-51

\title{
RELACIÓN ENTRE EL ESTRÉS PSICOLÓGICO Y LA PRESIÓN ARTERIAL MEDIA: UN ESTUDIO PILOTO DE LABORATORIO
}

\author{
David Alberto Rodríguez-Medina', Irving Armando Cruz-Albarrán², Gerardo Leija-Alva³ y Benjamín \\ Domínguez-Trejo 4 \\ Facultad de Psicología, UNAM ${ }^{1,4}$; Facultad de Ingeniería, UAQ ${ }^{3}$; Centro Interdisciplinario de Ciencias de la Salud, IPN² \\ México
}

\section{RESUMEN}

El objetivo de este estudio piloto fue explorar la relación entre la presión arterial media (PAM) y el estrés psicológico en un grupo de participantes clínicamente sanos (alimentación, conductas preventivas y valoración negativa ante eventos estresantes dentro de rangos normales). Para valorar su nivel de estrés, incluyendo los criterios de inclusión, se utilizó el Perfil de Estrés de Nowack. Posteriormente se administró a los participantes el Trier Social Stress Test (TSST) para explorar el efecto de este paradigma sobre la PAM. Los resultados mostraron una relación directa estadísticamente significativa entre la PAM y el nivel de estrés, y una relación inversa entre la PAM y el apoyo social. La PAM post TSST aumentó estadísticamente. Los resultados se analizan en el contexto de la utilidad de estas medidas clínicas para diseñar programas de intervención basados en evidencia y necesidades específicas con aplicaciones inmediatas en contextos universitarios para manejo de estrés.

Palabras Clave:

presión arterial media, apoyo social, Trier Social Stress Test.

\section{RELATIONSHIP BETWEEN PSYCHOLOGICAL STRESS AND MEAN ARTERIAL PRESSURE: A LABORATORY PILOT STUDY}

\begin{abstract}
The objective of this pilot study was to explore the relationship between mean arterial pressure (MAP) and psychological stress in a group of clinically healthy participants (feeding, preventive behaviors and negative assessment of stressors within normal ranges). In order to assess their stress level, including the inclusion criteria, we used the Nowack Stress Profile. Subsequently, Trier Social Stress Test (TSST) was administered to participants to explore the effect of this paradigm on MAP. The results showed a statistically significant direct relationship between MAP and the level of stress; and an inverse relationship between MAP and social support. Post-TSST MAP increased statistically. The results are discussed in the context of the utility of clinical measures to design intervention programs based on evidence and specific needs with immediate application in university contexts for stress management.
\end{abstract}

Keywords:

Mean Arterial Pressure, Social Support, Trier Social Stress Test..

BitÁCORA DEL ARTículo:

| Recibido: 19 de febrero de 2017 | Aceptado: 24 marzo de 2017 | Publicado en línea: enero-junio de 2017 | 


\title{
RELACIÓN ENTRE EL ESTRÉS PSICOLÓGICO Y LA PRESIÓN ARTERIAL MEDIA: UN ESTUDIO PILOTO DE LABORATORIO
}

\author{
David Alberto Rodríguez-Medina, Irving Armando Cruz-Albarrán, Gerardo Leija-Alva \\ y Benjamín Domínguez-Trejo
}

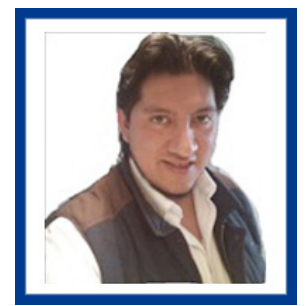

David Alberto Rodríguez Medina Faculta de Psicología, UNAM

Correo: psic.d.rodriguez@comunidad. unam.mx

Licenciado en Psicología por parte de la UNAM, con estudios especializados en Psicofisiología Aplicada a la Psicología Clínica y de la Salud por parte del INPRFM; actualmente realiza su doctorado en Psicología y la Salud en la Facultad de Psicología de la UNAM.

Ver más...

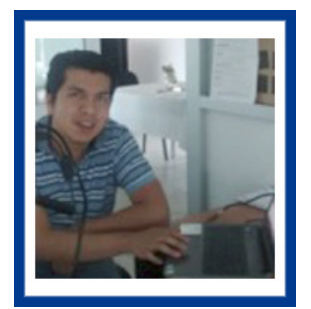

Irving Armando Cruz Albarrán Facultad de Ingenieria, UAQ Correo: icruz@hspdigital.org

Ingeniero electromecánico egresado del Tecnológico de Estudios Superiores de Jocotitlán en 2012. Actualmente es estudiante del doctorado en Mecatrónica en la Universidad Autónoma de Querétaro.

Ver más...

\section{CONTRIBUCión DE lOS Autores}

El primer autor desarrolló el proyecto de investigación, la investigación documental, capturó y anlisisó los datos. | Irving Armando Cruz-Albarran coordinó el de trabajo de campo, organizó la participación de los voluntarios, dió apoyo logístico y en el registro de mediciones autonómicas. | Gerardo Leija-Alva manejó la escala y calificación del perfil psicofisiológico de estrés. | El ultimo autor coordinó los recursos.

\section{AGRADECIMIENTOS}

El proyecto de investigación fue realizado con el apoyo del Proyecto PAPIME, Clave PE300716. Fronteras de la Psicología: Citocinas y emociones, y del Proyecto PAPIIT, Clave IN304515. Biomarcadores (autonómicos e inmunológicos) como indicadores del componente emocional en el dolor crónico.

\section{Datos de Filiación DE LS AUtores}

Facultad de Psicología, UNAM | Facultad de Ingeniería, UAQ | Centro Interdisciplinario de Ciencias de la Salud, IPN | Facultad de Psicología, UNAM

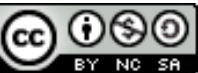

Copyright: @ 2017 Rodríguez-Medina, D.A., Cruz-Albarrán, I.A., Leija-Alva, G., \& Domínguez-Trejo, B.

Este es un artículo de acceso abierto distribuido bajo los términos de la licencia Creative Commons Reconocimiento-NoComercial-Compartirlgual 4.0 Internacional, por lo que su contenido gráfico y escrito se puede compartir, copiar y redistribuir total o parcialmente sin necesidad de permiso expreso de sus autoras con la única condición de que no se puede usar con fines directamente comerciales y los términos legales de cualquier trabajo derivado deben ser los mismos que se expresan en la presente declaración. La única condición es que se cite la fuente con referencia a la Revista Digital Internacional de Psicología y Ciencia Social y a sus autoras. 


\section{TABLA DE CONTENIDO}

INTRODUCCIÓN

MÉTOdO

Participantes, 4

Materiales, 5

Procedimiento, 5

Mediciones, 5

Análisis estadísticos, 5

RESULTADOS

Discusión

CONCLUSIONES

REFERENCIAS 


\section{INTRODUCCIÓN}

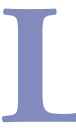

a evaluación del estrés ha sido uno de los pilares dentro de la Psicología de la Salud. Para medir el estrés existen diversas escalas psicométricas —medidas psicofisiológicas periféricas (respuestas cardiovasculares, tales como: tasa cardiaca, variabilidad de la frecuencia cardiaca, presión arterial, temperatura periférica-, respuestas somáticas - como la frecuencia respiratoria y la actividad electromiográfica, entre otras - y medidas psicofisiológicas centrales - la actividad electroencefalográfica- (Amigo, 2015). La selección de la medida adecuada para su evaluación depende del modelo psicológico: la teoría polivagal, para evaluar la actividad autonómica, o el enfoque cognitivo transaccional de Lazaruz (Nowack, 2002).

Dentro de las medidas psicológicas se encuentra el perfil de estrés de Nowack (2002), el cual permite evaluar aspectos físicos, como los hábitos de salud (ejercicio, sueño, alimentación, prevención) y aspectos psicológicos (apoyo social, personalidad tipo A, estilos de afrontamiento y bienestar psicológico). Este perfil cuenta con buenas propiedades psicométricas y valores normativos mediante puntuaciones $\mathrm{T}$, que permiten distinguir tres niveles por cada subescala: bajo, normal y alto. Su uso en México ha sido documentado en población universitaria (Preciado y Vázquez, 2010; Tafoya, Jurado, Yépez, Fouloux y Lara, 2013).

Debido a que existen diferentes factores que modulan el estrés (genético, biológico, psicológico y social), es necesaria la medición multifactorial. El Trier Social Stress Test (TSST) originalmente propuesto por Kirschbaum, Pirke y Hellhammer (1993) ha sido objeto de estudio de estrés social y se ha evaluado su efecto en medidas psicofisiológicas (como la presión arterial que se considera en la presente investigación), inmunológicas y endocrinológicas (Allen, Kennedy, Cryan, Dinan y Clarke, 2014). Sus versiones más recientes (Birkett, 2011; Birkett, Johnson y Gelety, 2016) consideran tres fases: 1) la condición prelínea base, donde el sujeto se ambienta al lugar de medición; 2) la línea base, donde se le miden respuestas psicofisiológicas y escalas psicológicas, y 3) el ensayo de estresor social, en el cual se proporcionan indicaciones al sujeto para preparar un discurso de porqué sería el candidato idóneo para su trabajo ideal (fase de preparación) durante tres minutos; luego se le pide que presente el discurso de manera verbal frente a una videocámara durante cinco minutos. Al término de los cinco minutos se instruye al sujeto a realizar una tarea aritmética mental progresiva donde el error descontinúa los avances, por lo que tiene que volver a empezar desde el inicio. Desde la preparación del discurso y hasta el término de la tarea aritmética se enciende la videocámara y se indica al sujeto que su discurso será analizado por un panel de expertos. Dependiendo de los objetivos del estudio, se puede considerar una cuarta fase para evaluar la recuperación en las medidas que el investigador esté interesado.

Lo que aún no está claro es si la presión arterial se incrementa, tanto sistólica como diastólica, o sólo una de ellas (Allen et al., 2014). Una medida que contempla ambas presiones es la presión arterial media, que puede definirse como la suma de la presión sistólica y dos veces la presión diastólica, y esta suma dividida entre tres. Dado que se conocen los valores óptimos de la presión arterial, es posible ubicar esta presión arterial media en los rangos establecidos por la norma oficial mexicana de acuerdo con la edad e índice de masa corporal (Secretaría de Salud, 2012). El presente estudio tiene como objetivo principal explorar las relaciones entre la presión arterial media en un estado de reposo y los niveles categóricos bajo, normal y alto de acuerdo con las puntuaciones T del perfil de estrés de Nowack en población clínicamente sana: alimentación adecuada, con actividad física regular de ejercicio, sin problemas de sueño y sin conductas adictivas, como el tabaquismo, a fin de ponderar el estrés psicológico y apoyo social sobre la presión arterial media.

El objetivo secundario es explorar los cambios en la presión arterial media pre-post del paradigma TSST en un grupo de estudiantes universitarios clínicamente sanos.

\section{MÉTodo}

\section{Participantes}

Se obtuvo un muestreo no probabilístico, por conveniencia, de 27 estudiantes universitarios voluntarios. Para este estudio se consideraron los participantes con un índice de masa corporal $<25$, con niveles normales de puntuación del perfil de estrés de Nowack en las subescalas físicas de alimentación y prevención, así como un nivel de puntuación normal en la subescala psicológica de valoración negativa. Estos criterios de inclusión/exclusión permitieron depurar factores físicos (obesidad) y subjetivos (pensamientos catastróficos) que expliquen la PAM en estado de reposo. La muestra final quedó conformada por cinco estudiantes masculinos universitarios, edad media $=24.6$ años, clínicamente sanos, sin historial de enfermedades psiquiátricas, neurológicas o crónicas (transmisibles y no transmisibles). 


\section{Materiales}

Perfil de estrés de Nowack (2002). Es un instrumento autoaplicable que evalúa áreas relacionadas con el estrés psicológico mediante 123 reactivos agrupados en 15 áreas, y éstas se distribuyen en factores conductuales de hábitos de salud, factores cognitivos y subjetivos. Cuenta con buenas propiedades psicométricas de validez, confiabilidad y estandarización para población general de 20 a 68 años. Establece puntos de corte para identificar tres niveles de cada subescala: bajo (T20-T39), normal (T40-T59) y alto (T60-T80).

Videocámara digital Canon ZR850. Dispositivo de uso comercial para la videograbación del discurso y la tarea aritmética.

Baumanómetro digital CITIZEN CH-656 C. Equipo electrónico comercial para evaluar la presión arterial, cuya calidad en la medición clínica ha sido probada (Cotte, Faltenbacher, von Willich y Bogner, 2008). Este equipo se utilizó de acuerdo con las instrucciones del fabricante (sentado, apoyando el brazo izquierdo con el codo en una mesa y flexionando el antebrazo con la muñeca a la altura del corazón). El manual del equipo sostiene que el sujeto debe permanecer al menos 10 minutos sentado. Para propósitos de esta investigación se utilizó la presión arterial media, que contempla la presión arterial sistólica y diastólica, cuya fórmula para su cálculo es:

$$
\mathrm{PAM}=2 / 3(\mathrm{PAD})+1 / 3(\mathrm{PAS})
$$

Donde:

- PAM: presión arterial media.

- PAD: presión arterial diastólica.

- PAS: presión arterial sistólica.

Todos los registros fueron tomados en aulas universitarias iluminadas, con control de temperatura ambiental, asientos y mesa de trabajo para apoyar el brazo y tomar la presión arterial, así como para la aplicación de la prueba psicológica del perfil de estrés y el TSST.

\section{Procedimiento}

Al llegar los participantes al aula firmaron el consentimiento informado. Con base en el protocolo adaptado de Birkett (2011) se replicó el TSST (figura 1): primero, al llegar los participantes al laboratorio se les brindó el consentimiento informado. A continuación se aplicó el perfil de estrés de Nowack; 20 minutos después de llegar se les tomó la presión arterial y se calculó la PAM pre-test. Finalmente, en un salón contiguo al de la administración del perfil de estrés se aplicó el TSST y, al término del mismo, se volvió a registrar su presión arterial y se obtuvo su PAM post-test.

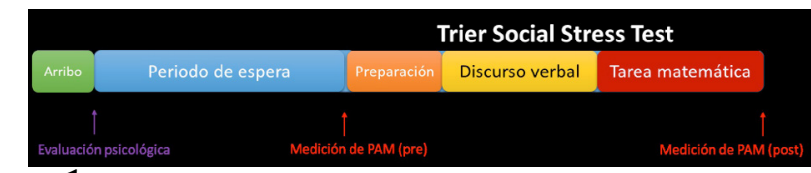

Figura 1.

Diseño de investigación pre-experimental del Trier Social Stress Test.

\section{Mediciones}

Se utilizó un diseño mixto: transversal para la evaluación de las puntuaciones del perfil de estrés de Nowack. Para la medición de la PAM, se utilizó un diseño prepost TSST. La medición pre-test se relacionó con los niveles de las puntuaciones T del perfil de estrés.

\section{Análisis estadísticos}

Debido al tamaño de la muestra se optó por utilizar estadística no paramétrica para el análisis de los datos. Para el análisis de la PAM pre-post TSST se utilizó Wilcoxon. Para evaluar la relación entre la PAM y la puntuación T de las subescalas de estrés psicológico se utilizó un estadístico rho de Spearman. Se estableció una significancia estadística de alfa $\leq 0.05$.

\section{Resultados}

Los datos descriptivos de las características sociodemográficas de la muestra se presentan en la tabla 1.

Tabla 1

Datos sociodemográficos de la muestra.

\begin{tabular}{cc}
\multicolumn{2}{c}{ Datos Sociodemográficos } \\
Sujetos (N) & 5 \\
Sexo & Masculino \\
Edad (Media) & 24.6 años \\
Escolaridad (años) & $17-19$ años \\
Ocupación & Estudiantes de posgrado en \\
Estado civil & Ingeniería \\
Índice de masa corporal & Soltero ( $\mathrm{N}=5)$ \\
\end{tabular}

Las características psicológicas del perfil de estrés y su PAM pre-test de cada participante se muestran en la tabla 2.

\section{Tabla 2.}

Características psicológicas de los participantes y su presión arterial media (PAM). 
Existe una relación directa entre el estrés psicológico y la PAM, y una relación inversa entre el apoyo social y la PAM. Estas fueron las únicas dos variables psicológicas que mostraron relación con la actividad autonómica. Las figuras 2 y 3 muestran las relaciones estadísticamente significativas de la PAM con los niveles de estrés percibido y la red de apoyo social respectivamente. Entre los niveles de estrés y de apoyo social se encontró una alta correlación estadísticamente significativa $(r=-0.913, p=0.003)$.

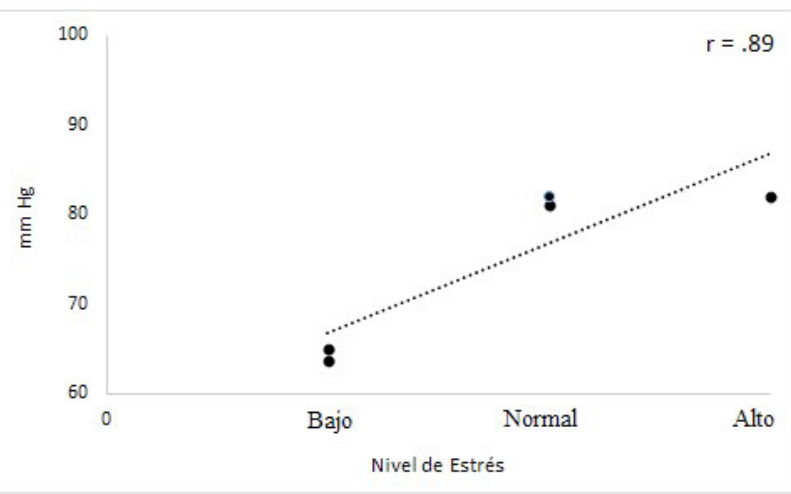

Figura 2.

Relación entre el nivel de estrés y la PAM

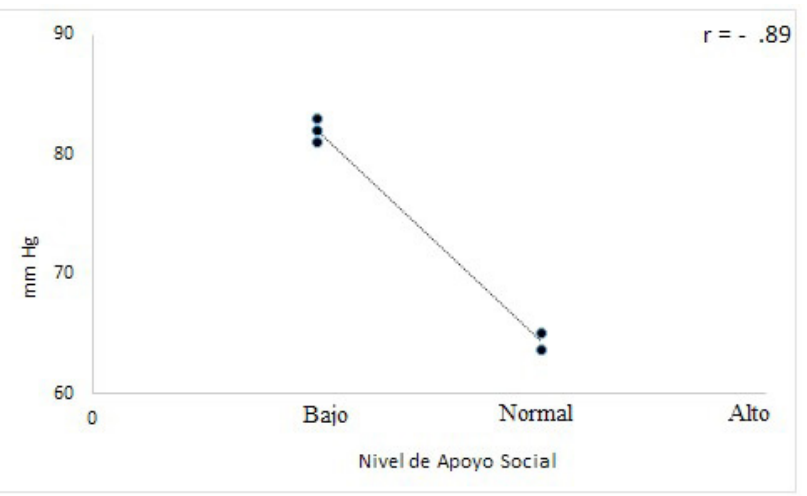

Figura 3.

Relación entre el nivel de apoyo social y la presión arterial media

Además, se encontraron correlaciones estadísticamente significativas entre los índices $T$ del perfil de estrés entre: PAM y el puntaje de alimentación $(r=-0.892, p=$ $0.042)$; hábitos de salud con el de prevención $(r=.9, p=$ 0.037); los índices $T$ de prevención con minimización de la amenaza $(r=0.975, p=0.005)$ y con la concentración en el problema $(r=-0.9, p=0.037)$; el conglomerado ARC con la valoración positiva $(r=-0.892, p=0.042)$; la conducta tipo A y el bienestar psicológico $(r=-0.894, p$ $=0.041$ ) y la concentración en el problema y la minimización de la amenaza $(r=-0.975, p=0.005)$.
Los datos de la PAM pre-post TSST indican un incremento estadísticamente significativo en las medianas (figura 4).

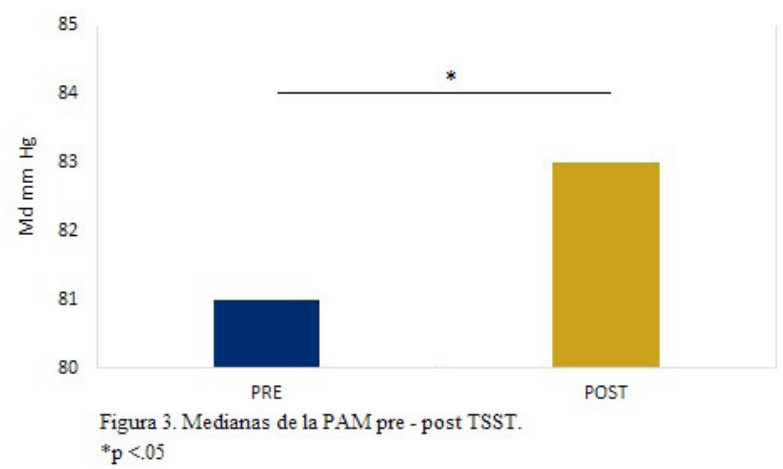

Figura 4.

Medianas de los cambios de la PAM pre - post TSST.

\section{Discusión}

Los resultados obtenidos en el presente estudio permiten una lectura general del estado de salud de los jóvenes universitarios. El primer dato que surge es el tamaño de la muestra que cumple con características saludables; apenas $18.5 \%$ de la muestra cumplió con los criterios de inclusión. Este dato sugiere estimar las características físicas y psicológicas de la población estudiantil universitaria.

En esta investigación se evaluó la relación entre la PAM y las puntuaciones del perfil de estrés de Nowack en una muestra con hábitos de salud normales. Con los criterios de inclusión y exclusión destaca la estrecha relación de la PAM con el nivel de estrés y el apoyo social. El nivel de apoyo social se ha documentado en diversas publicaciones epidemiológicas y psicológicas como un mediador de estrés (Amigo, 2015) con un sólido nivel de evidencia experimental psicobiológica (Wittig, Crockford, Weltring, Langergraber, Deschner y Zuberbühler, 2016) en la regulación hormonal de la actividad endócrina. En la investigación clínica se ha documentado la importancia de construir redes de apoyo social. Por ejemplo, en un padecimiento como el dolor crónico, modula la actividad inflamatoria, la sintomatología depresiva y el nivel de dolor percibido (Hughes et al., 2014). Es probable que en la población con estas características de hábitos de salud como apoyo social maneje mejor el estrés y sus efectos en la presión arterial respecto a la población que carece de estas cualidades.

La PAM, medida con el equipo de uso comercial, fue sensible a los cambios autonómicos del TSST; sin embargo, en uno de los sujetos evaluados no incrementó la PAM, sino al contrario, decrementó. Esto coincide con su punta- 
je T del perfil de estrés de afrontar el problema: bajo nivel de concentración en el problema. Este resultado indica que los instrumentos de medición empleados son útiles tanto para la investigación como para la práctica clínica.

El alcance de esta investigación piloto coincide con los reportaros por Allen et al. (2014). La PAM, que utiliza la combinación de la presión sistólica y la presión diastólica, puede ser considerada como una medida sensible a la evaluación del TSST, en lugar de sólo evaluar una de ellas. Queda pendiente extender el periodo de registro psicofisiológico a una cuarta fase de recuperación de la PAM para evaluar la respuesta adaptativa del estrés agudo generado por el TSST y su utilidad en el contexto clínico, como los pacientes con vulnerabilidad a problemas cardiovasculares. De esta manera se podría replicar esta investigación con un aumento en el tamaño de la muestra y con la implementación de medidas autonómicas periféricas adicionales, como la variabilidad de la frecuencia cardiaca o la imagen infrarroja térmica (Ioannou, Gallese y Merla, 2014; Domínguez, Rodríguez, Pluma, Cruz y Morales, 2016; Rodríguez y Domínguez, 2016) que soporten un modelo conceptual y estadístico del peso de los aspectos psicológicos sobre la presión arterial media en periodos de estrés agudo.

\section{Conclusiones}

La relación entre el nivel de estrés y la PAM reportado en este grupo permite valorar el aspecto psicológico sobre una medida fisiológica, incluso prescindiendo de los participantes con un nivel alto de valoración negativa en quienes se ha documentado mayor vulnerabilidad a trastornos emocionales. Esta relación entre la PAM y el nivel de estrés percibido sugiere la réplica con un tamaño de muestra con el objetivo de generar programas universitarios de promoción de la salud interdisciplinarios (enfermería, psicología, medicina interna, cuando menos en las universidades que cuentan con estos servicios) de monitoreo continuo para la vigilancia y manejo clínico de la PAM en caso necesario; por ejemplo, para las personas con antecedentes de enfermedades crónicas, como el sobrepeso, obesidad, adicciones, hipo e hipertensión arterial. Eventualmente se podrían aplicar estrategias psicológicas para la regulación de la actividad autonómi$\mathrm{ca}$, como ya se ha probado en otras investigaciones, incluso en México, para el manejo de cuadros clínicos como la hipertensión arterial (Alcazar, Riveros, Sánchez y Cortazar, 2005). De acuerdo con el perfil de estrés de Nowack y la implementación de un perfil psicofisiológico de estrés monitoreado con instrumentos tecnológicos de bajo costo, como termómetros digitales o equipos de termografía desarrollados en México, se puede optar por realizar intervenciones psicológicas cognitivo-conductuales, cuando los problemas son conductas adictivas y valoración negativa; estrategias psicofisiológicas, cuando posean alta reactividad al estrés y no permita una pronta recuperación del balance autonómico; $y / o$ intervenciones psicosociales, cuando se trate de manejar conflictos afectivos y sociales para reducir los niveles de estrés y su actividad autonómica asociada.

\section{Referencias}

Alcazar L., Riveros, A., Sánchez-Sosa, J J. y Cortazar-Palapa, J., (2005). Efectos de una intervención cognitivo-conductual en la calidad de vida, ansiedad, depresión y condición médica de pacientes diabéticos e hipertensos esenciales. International Journal of Clinical and Health Psychology, (5) 445-462. Recuperado de http://www.redalyc.org/articulo. oa?id=33705302

Allen, A., Kennedy, P., Cryan, J., Dinan, T., y Clarke, G. (2014). Biological and psychological markers of stress in humans: Focus on the Trier Social Stress Test. Neuroscience y Biobehavioral Reviews, 38, 94-124. http://dx.doi. org/10.1016/j.neubiorev.2013.11.005

Amigo, I. (2015). Manual de psicología de la salud (2nd ed., pp. 111 - 115). Madrid: Ediciones Pirámide.

Birkett, M. (2011). The Trier Social Stress Test Protocol for Inducing Psychological Stress. Journal Of Visualized Experiments, (56). http://dx.doi.org/10.3791/3238

Birkett, M., Johnson, L., y Gelety, C. (2016). Investigation of Sex Differences In slgA Response to the Trier Social Stress Test. Stress And Health. http://dx.doi.org/10.1002/smi.2680

Cotte, U., Faltenbacher, V., von Willich, W., y Bogner, J. (2008). Trier of validation of two devices for self-measurement of blood pressure according to the European Society of Hypertension International Protocol: the Citizen $\mathrm{CH}-432 \mathrm{~B}$ and the Citizen $\mathrm{CH}-656 \mathrm{C}$. Blood Pressure Monitoring, 13(1), 55-62. http://dx.doi.org/10.1097/ mbp.0b013e3282cb599a

Domínguez, B., Rodríguez, D., Pluma, S. Cruz, I., Morales, L. (2016). Respuesta Inflamatoria, emociones y dolor. Investigación clínica. Lecture, Seminario de Neurociencias, División de Ciencias Biológicas y de la Salud, Universidad Autónoma Metropolitana, Unidad Iztapalapa. http://doi:10.13140/ RG.2.1.4417.3044

Hughes, S., Jaremka, L., Alfano, C., Glaser, R., Povoski, S., y Lipari, A. etal.(2014). Social support predicts inflammation, pain, and depressive symptoms: Longitudinal relationships among breast cáncer survivors. Psychoneuroendocrinology, 42, 38-44. http://dx.doi.org/10.1016/j.psyneuen.2013.12.016

Ioannou, S., Gallese, V., y Merla, A. (2014). Thermal infrared imaging in psychophysiology: Potentialities and limits. Psychophysiology, 51(10), 951-963. http://dx.doi. org/10.1111/psyp.12243

Kirschbaum C, Pirke KM, Hellhammer DH (1993) The 'Trier Social Stress Test' - a tool for investigating psychobiology stress responses in a laboratory setting. Neuropsychobiol 28:76-81 http://dx.doi.org/10.1159/000119004

Nowack K. (2002). Perfil de Estrés. México: Manual Moderno. 
Preciado, M. y Vázquez, J. (2010). Perfil de estrés y síndrome de burnout en estudiantes mexicanos de odontología de una universidad pública. Revista Chilena De NeuroPsiquiatría, 48(1), 11-19. http://dx.doi.org/10.4067/S071792272010000200002

Rodríguez, D. y Domínguez, B. (2016). Perfil biopsicosocial del dolor crónico oncológico y efectos térmicos en la imagen infrarroja de la expresión facial emocional. In M. Padilla Vargas, S. Galán Cuevas, E. Camacho Gutiérrez y A. Zárate Loyola, Investigación en Psicología básica y aplicada: avances y perspectivas (1st ed., pp. 498-503). Universidad Autónoma de San Luis Potosí. San Luis Potosí: Orgánica Editores. Recuperado de http://www.organicaeditores. $\mathrm{mx} /$ biblioteca/?bookid=77

Secretaria De Salud, (2012). I. Resumen integrado Norma Oficial Mexicana NOM-030-SSA2-2009, para la prevención, detección, diagnóstico, tratamiento y control de la hipertensión arterial sistémica. Revista Mexicana De Cardiología, 23(Suplemento 1), 4a - 38a. Recuperado de http://www.medigraphic.com/pdfs/cardio/h-2012/ hs121a.pdf
Tafoya, S., Jurado, M., Yépez, N., Fouloux, M., y Lara, M. (2013). Los hábitos de salud como posibles protectores de dificultades en el sueño en estudiantes de medicina de una universidad nacional de México. Anales de la Facultad De Medicina, 74(3), 187 -92. http://dx.doi.org/10.15381/ anales.v74i3.2633

Wittig, R., Crockford, C., Weltring, A., Langergraber, K., Deschner, T., y Zuberbühler, K. (2016). Social support reduces stress hormone levels in wild chimpanzees across stressful events and everyday affiliations. Nature Communications, 7, 13361. http://dx.doi.org/10.1038/ncomms13361 


\section{Meta-Análisis del Artículo}




\section{Dimensión Cuantitativa}

\section{Perfil de Evaluación entre pares}
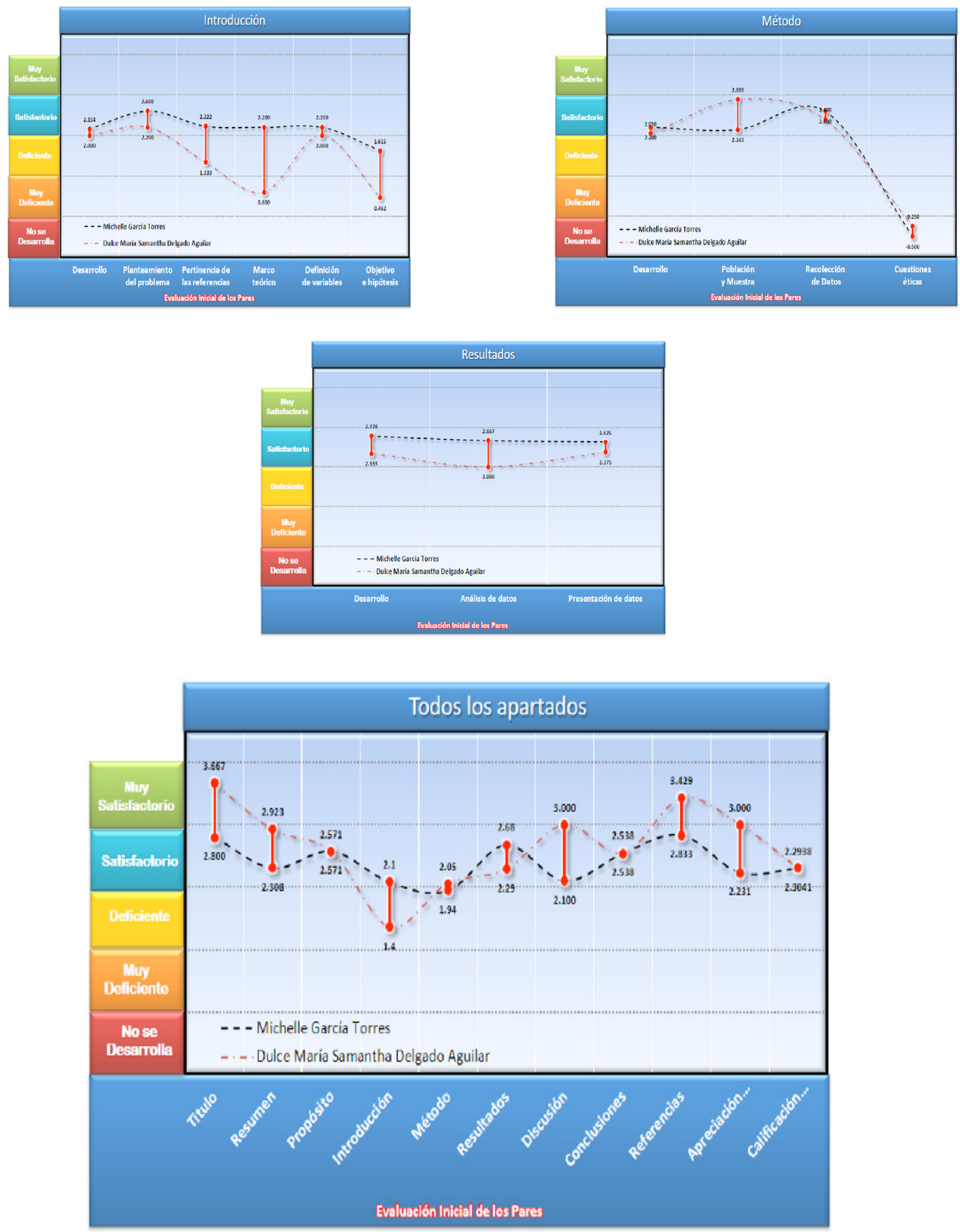
Revista Digital Internacional de Psicología y Ciencia Social |Vol. 3 | Nóm. 1 | Enero-Junio 2017| ISSN 2448-8119 e-ISSN $2448-8119$

\section{Índice de Concordancia}

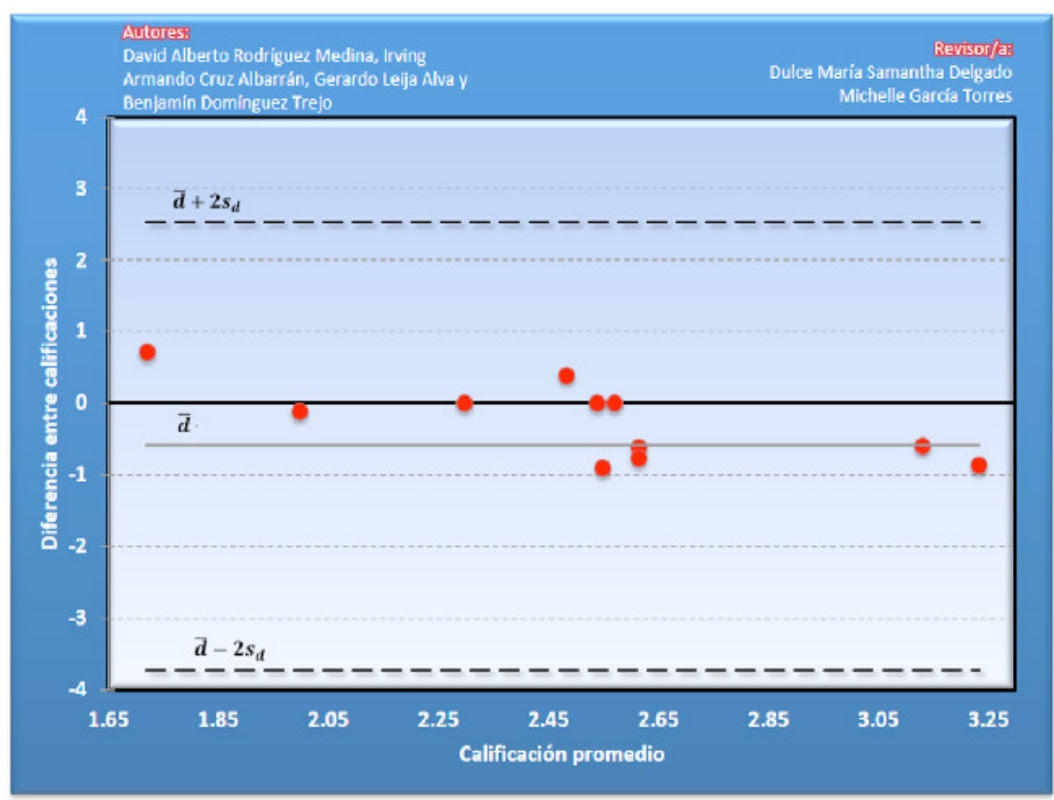

Índice de Acuerdo

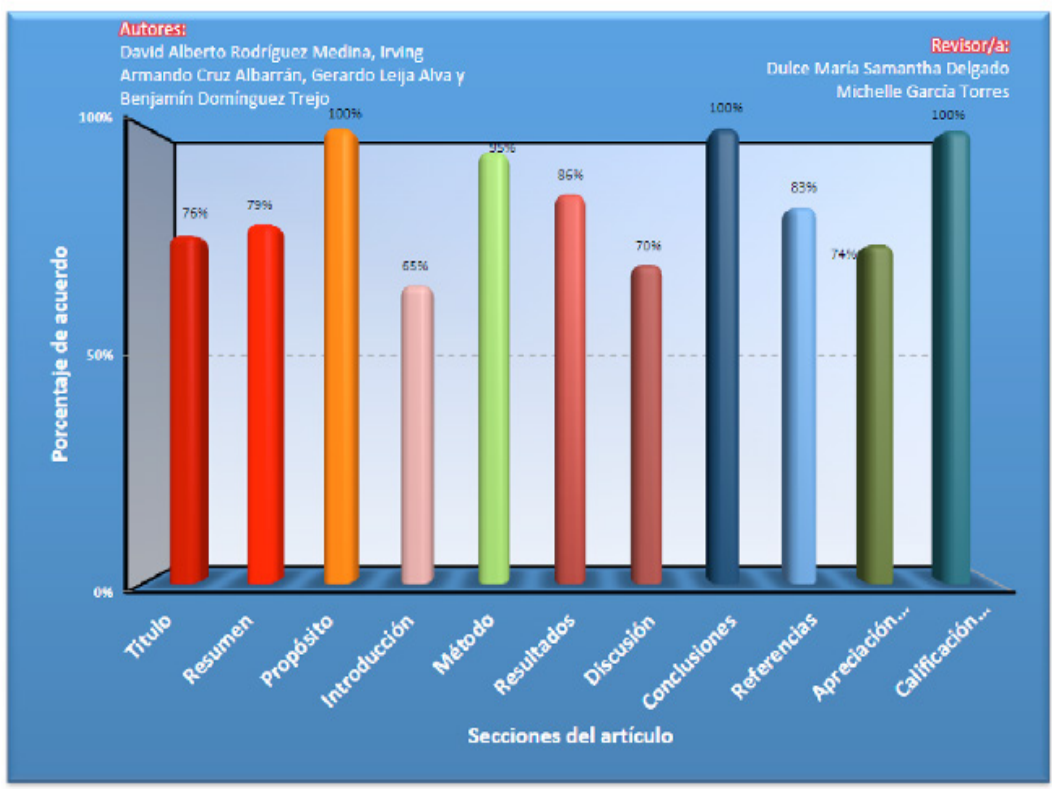


Dimensión Cualitativa

\begin{tabular}{|c|c|}
\hline Revisor 1 & Revisor 2 \\
\hline Michelle García Torres & Dulce María Samantha Delgado Aguilar \\
\hline
\end{tabular}

\section{Título/Autoría}

El título es adecuado y claro.

Se deben anexar los correos de dos autores restantes y omitir los títulos académicos.

\section{Resumen}

La metodología es confusa cuando se describe la medición de los criterios de inclusión y la medición de la variable independiente Sería adecuado presentar resultados numéricos El resumen en inglés omite palabras importantes presentes en la versión en Español.
Se debe especificar la metodología seguida, así como los resultados más relevantes del estudio en forma de valores numéricos y replantear la discusión ya que no queda del todo claro la trascendencia del estudio.

\section{Próposito del Estudio}

Sería recomendable que los autores presentaran hipótesis de trabajo si es que se plantearon de antemano.
Hace falta relacionar de manera más clara, breve y concisa los objetivos a seguir y los resultados, además se debe explicitar si estos fueron aceptados o refutados de acuerdo a sus resultados.

\section{Introducción}

Es recomendable que se conozcan las hipótesis que los autores tenían antes de realizar el estudio, así como lo encontrado sobre dicho tema por otros investigadores.
En necesario incluir las referencias revisadas para dar sustento a la investigación actual y resaltar cuál es el propósito por la que se llevó a cabo así como de vislumbrar de qué manera se pretende aportar a la resolución del problema; para esto es completamente necesario aclarar las variables que se medirán, cómo se medirán y de qué forma están relacionadas, para que al final, los objetivos sean más comprensibles y concuerden con la hipótesis planteada, que debe estar señalada explícitamente. 
Revista Digital Internacional de Psicología y Ciencia Social |Vol. 3 | Núm. 1 | Enero-Junio 2017| ISSN 2448-8119 e-ISSN 2448 -8119

\begin{tabular}{|c|c|}
\hline Revisor 1 & Revisor 2 \\
\hline \multicolumn{2}{|c|}{ Método } \\
\hline $\begin{array}{l}\text { Se recomienda describir la muestra con la que se trabajó, } \\
\text { su obtención, relevancia y trato ético brindado }\end{array}$ & $\begin{array}{l}\text { El desarrollo del diseño y recolección de datos debe ir } \\
\text { descrito paso a paso para que los lectores entiendan } \\
\text { cada una de las fases y sesiones, su duración y lo que se } \\
\text { hizo en cada una de ellas y con qué se trabajó (pruebas } \\
\text { físicas o psicométricas) En la sección de participantes } \\
\text { se debe aclarar cómo se realizó la selección/evaluación } \\
\text { de los factores subjetivos. }\end{array}$ \\
\hline
\end{tabular}

Resultados

En el apartado de resultados se menciona la medición de aspectos que no se mencionan a lo largo del texto, como en el apartado de instrumentos Algunas tablas son difíciles de interpretar.
Los resultados no están comprensibles, sólo se describen si hay o no correlación entre variables, pero no se explica la importancia de éstos resultados, por lo que se debe ahondar en la interpretación de los mismos. Incluir descripción a figuras, tablas o gráficas.

\section{Discusión}

Se sugiere citar estudios en los que se hayan encontrado cosas similares o incluso diferentes Los trabajo citados en la discusión refieren a estudios en los que realizaron cosas similares más no se abordan los resultados, eso Cuidar la manera en que se cita a los autores y la ortografía.

\section{Conclusiones}

Remarcar la relevancia de los resultados obtenidos y su utilidad inmediata.

Se debe escribir cuáles son los aportes del estudio a la problemática así como los problemas de implementación así como alternativas de abordaje al problema central de la investigación.

\section{Referencias}

Se recomienda revisar el manual de la APA para que las referencias cumplan con los requisitos indispensables.
Incluir todas las referencias como citas y reescribirlas de acuerdo al APA. 
